\title{
Personalien
}

Praxiseröffnung /

Nouveaux cabinets médicaux /

Nuovi studi medici

BE

\section{Romain Zahnd,}

Facharzt für Endokrinologie/Diabetologie und Facharzt für Allgemeine Innere Medizin, Hirschengraben 10, 3011 Bern

\section{FR}

\section{Mickaël Ludovic Ransan,}

Médecin praticien, 76, Grand-Rue, 1627 Vaulruz

Ray Leda Cilacian,

Spécialiste en médecine interne générale, 4, avenue de la Gare, 1700 Fribourg

\section{VS}

Olivier Marc André Heldenbergh,

Spécialiste en ophtalmologie, 3, rue du Bourg, 3960 Sierre

Marie Ludvine Delarge,

Spécialiste en psychiatrie et psychothérapie, 43, rue de Lausanne, 1950 Sion

\section{Aargauischer Ärzteverband}

Zur Aufnahme in den Aargauischen Ärzteverband haben sich angemeldet:

als ordentlich praktizierende Mitglieder:

Niclas Freund, Facharzt für Innere Medizin und Facharzt für Kardiologie, 4056 Basel, Praxiseröffnung in Praxisgemeinschaft in Rheinfelden per 1. September 2015

Bernd Heinrich, Facharzt für Orthopädische Chirurgie und Traumatologie des Bewegungsapparates, D-88212 Ravensburg, Praxiseröffnung in Praxisgemeinschaft in Reinach per 1. September 2015
Andrea Hermatschweiler, Fachärztin für Allgemeine Innere Medizin FMH, 4310 Rheinfelden, Angestellt in Praxisgemeinschaft in Rheinfelden seit 1. Mai 2015

als Chef- und Leitende ÄrztInnen

Angelika Hammerer-Lercher, A-6073 Sistrans, Leitende Ärztin im Kantonsspital Aarau in Aarau per 1. September 2015

Leonhard Schäffer, Facharzt für Gynäkologie und Geburtshilfe, 5442 Fislisbach, Chefarzt im Kantonsspital Baden AG in Baden seit 1. Juli 2014

Jörg-Peter Sigle, Facharzt für Innere Medizin und Facharzt für Hämatologie, 4053 Basel, Chefarzt in der Stiftung Blutspende SRK Aargau-Solothurn in Aarau seit 1. Mai 2015

Diese Kandidaturen werden in Anwendung von Art. 5 der Statuten des Aargauischen Ärzteverbandes veröffentlicht. Einsprachen müssen innert 14 Tagen seit der Bekanntmachung schriftlich und begründet der Geschäftsleitung des Aargauischen Ärzteverbandes eingereicht werden. Nach Ablauf der Einsprachefrist entscheidet die Geschäftsleitung über Gesuch und allfällige Einsprachen.

Ärztegesellschaft des Kantons Bern Ärztlicher Bezirksverein Bern Regio Zur Aufnahme als ordentliches Mitglied hat sich angemeldet:

Jörg Hummen, Facharzt für Psychiatrie und Psychotherapie, Giessereiweg 9, 3007 Bern

Einsprachen gegen dieses Vorhaben müssen innerhalb 14 Tagen seit der Veröffentlichung schriftlich und begründet beim Präsidenten des Ärztlichen Bezirksvereins Bern Regio eingereicht werden. Nach Ablauf der Frist entscheidet der Vorstand über die Aufnahme der Gesuche und über die allfälligen Einsprachen.
Ärztegesellschaft des Kantons Luzern

Zur Aufnahme in unsere Gesellschaft Sektion Stadt hat sich gemeldet:

Leila Bichsel, Fachärztin für Allgemeine Innere Medizin FMH, Dufourstrasse 12, 6003 Luzern

Zur Aufnahme in unsere Gesellschaft Sektion Gäu haben sich gemeldet:

Michael Bode, Facharzt für Ophthalmologie, Argus Augen AG, Bahnhofstrasse 40, 6210 Sursee

Wjatscheslaw Akusok, Praktischer Arzt, Praxis ab 17.8.15: Gartenweg 5, 6252 Dagmersellen

Zur Aufnahme in unsere Gesellschaft Sektion Entlebuch hat sich gemeldet:

Vasile-Gheorghe Maghiar-Labai,

Praktischer Arzt, Xundheitszentrum Escholzmatt-Marbach, Bahnhofstrasse 11, 6182 Escholzmatt

Einsprachen sind innert 20 Tagen nach der Publikation schriftlich und begründet zu richten an: Ärztegesellschaft des Kantons Luzern, Schwanenplatz 7, 6004 Luzern

Ärzte-Gesellschaft des Kantons Zug Zur Aufnahme in die Ärzte-Gesellschaft des Kantons Zug als ordentliches Mitglied haben sich angemeldet:

Daniel Boller, Facharzt für Allgemeine Innere Medizin und Facharzt für Gastroenterologie, Baarerstrasse 11, 6300 Zug

Beat Unternährer, Facharzt für Allgemeine Innere Medizin, Bösch 37, 6331 Hünenberg

Einsprachen gegen diese Kandidatur müssen innerhalb 14 Tagen seit dieser Veröffentlichung schriftlich und begründet beim Sekretariat der Ärzte-Gesellschaft des Kantons Zug eingereicht werden. Nach Ablauf der Einsprachefrist entscheidet der Vorstand über Gesuch und allfällige Einsprachen. 